\title{
Studies regarding the use of a neuro-fuzzy mathematical model in order to determine the technological parameters of the polyethylene pipes butt welding process
}

\author{
Alina Gligor ${ }^{1, *}$, Marcel-Letitiu Balan ${ }^{2}$, Letitia-Corina Balan ${ }^{1}$, and Horatiu-Daniel Crisan ${ }^{3}$ \\ ${ }^{1}$ Lucian Blaga University of Sibiu, Faculty of Engineering, 4 E. Cioran St, 55025, Sibiu, Romania \\ ${ }^{2}$ E-on Gaz, 83 Stadionului st., 551105, Medias, Romania \\ ${ }^{3}$ Romgaz, 4, P-ta C.I. Motas, 551130, Medias, Romania
}

\begin{abstract}
The paper analyzes the possibility to use a neuro-fuzzy type mathematical model, with the final goal of establishing the welding parameters for new types and dimensions of pipes and fittings. Anticipating the developing dynamic of polyethylene-made elements, especially pipes and fittings, starting from the current situation when already a wide range of pipes and fittings with different wall thicknesses and nominal working pressures is produced and commercialized, and taking into account also new development, it was considered necessary to find out the welding parameters for any new pipe type and dimension. The usage of existing welding equipment for new pipe dimensions is impossible without a preliminary set of welding parameters: pressure, temperature, time. Based on experimentally validated data for discreet values of the characteristic welding parameters, there was generated, using mathematical laws and functions, a new model that can estimate the necessary values of the welding parameters for any value within their variation range. As a result, the mathematical model created using neuro-fuzzy techniques allows the obtaining of the correct value for certain parameters (e.g. required welding pressure) for any values of the input variables pipe diameter and pipe thickness.
\end{abstract}

\section{General aspects}

The paper analyzes the possibility to use a neuro-fuzzy type mathematical model, i.e. a logical system that is an extension of the multi-criteria evaluation logic, with the final goal of determining the welding parameters for new types and dimensions of pipes and fittings.

Anticipating the developing dynamic of the polyethylene materials, especially pipes and fittings, starting from the state of the art when already a wide range of pipes and fittings with different dimensions, wall thicknesses, nominal working pressure are produced and commercialized and looking towards new ones (intermediate diameters with specific wall thicknesses), it was considered necessary to find out the welding parameters for any new pipe type and dimension.

Using the existing welding equipment for new pipe dimensions becomes impossible without having a preliminary setup of the welding parameters: pressure, temperature, time.

* Corresponding author: alina.gligor@ulbsibiu.ro 
Based on some experimentally validated data for some discreet values of the characteristic parameters of the welding process, there was elaborated, using mathematical laws and functions that are implemented in the software system, a new model which can estimate the necessary values of the welding parameters for any value from their variation range, more precisely the values for the equipment setup, starting from the known and existing values.

The result is that that the mathematical model realized using neuro-fuzzy techniques gives the possibility to obtain the correct value for some parameters (e.g. welding pressure required) for any values of the input variables: pipe diameter and pipe thickness.

\section{Construction steps of a preliminary fuzzy model}

As was previously mentioned, the authors attempted to analyze the possibility of using fuzzy logic methods for the elaboration of a mathematical model regarding the butt welding process of the polyethylene pipes.

The advantages of using this method are:

- the fuzzy logic allows to obtain precise conclusions, based on poor, unprecise entry data (information);

- in the elaboration of the multicriterial fuzzy model, especially for the fuzzy type rule set, the experience of field professionals can be beneficial; this experience can successfully replace the use of highly complex mathematical algorithms;

- the fuzzy model has a high flexibility, resulting in its changing and refining being made with ease;

- both the gathering of information necessary for the application of the multicriterial decision fuzzy model and its usage can be done in a very short time, it can be described even as a real-time decision;

- nowadays there exists a large range of software programs that make the deployment of models based on fuzzy logic possible, very easily, without the need of advanced programming knowledge.

The elaboration of a fuzzy model requires going through the stages that will be presented below.

\section{Application of the Fuzzy Logic Methods}

The next step in approaching the problem is the application of the fuzzy logic methods for the data related to the existing input [1]. It should be taken into account that the structure of the mathematical model governing this problem is not known and one of the goals of this research is in fact to determine this model.

The fuzzy approach is complicated by the fact that it is difficult to establish the form of the affiliation functions for the proposed fuzzy model.

Most industrial processes have a multidimensional character. The mathematical modeling of these processes is very difficult, and in many cases impossible due to the absence of necessary information about the involved processes and about the interconnections between the components. Often, the information about processes are just examples of numerical data and linguistic forms of rules.

One of the methods for eliminating those difficulties is the use of both the theory of the fuzzy sets and of the artificial neuronal networks [2]. By applying the neuronal networks methods for the weak points of the fuzzy control and at the same time, the facilities of the fuzzy sets for the problems of the neuronal networks, a synthesis of both methods has been achieved. The method of the neuro-fuzzy intelligent shaping is a combination of the best performances of fuzzy logic and of the artificial neuronal networks. 
The basic idea of this approach is to use the techniques for the fuzzy model to learn, based on initial data input/output, in such a way as to calculate optimal affiliation functions, expressing the best interdependence of the input and output data.

The most important features of the theory of the fuzzy sets and artificial neuronal networks, for the purpose of their use in models of industrial processes, are shown in table 1.

Table 1. Characteristics of the fuzzy sets theory and of artificial neuronal networks for use in industrial process models.

\begin{tabular}{|c|l|l|}
\hline CHARACTERISTICS & \multicolumn{1}{|c|}{ NEURO } & \multicolumn{1}{c|}{ FUZZY } \\
\hline Advantages & $\begin{array}{l}\text { - learning possibility based on numerical } \\
\text { data files and generalization of the gained } \\
\text { knowledge }\end{array}$ & \\
\hline Disadvantages & - convergence of the learning process & $\begin{array}{l}\text { - absence of the functioning } \\
\text { adaptation of components }\end{array}$ \\
\hline
\end{tabular}

In a typical neuro-fuzzy structure following parts can be clearly distinguished:

- The fuzzy component, that consists in converting the value of a variable or quantitative values in a proper representation of the fuzzy sets;

- The artificial neuronal network component;

- The unfuzzyfication component that, using the method of the center of gravity or other methods, converts the outputs that the neuronal network expressed through appropriate fuzzy sets, into a simple numerical form.

\subsection{The construction of the mathematical model for the process}

The method studies the construction of a mathematical model for the studied process, based on the experimental data. The multivariable input character of the process and the fact that the material is exposed to some transformations allow to determine some analytical relations that characterize the process [3].

The goal is to build, based on experimentally validated data, for certain discrete values of the process parameters and using advanced modeling techniques, a model for estimating the required values of the parameters for any value within their variation range.

\subsubsection{Steps in the model construction}

A. In a first stage an attempt was made to construct a fuzzy model, but the complexity of the requirement to achieve this by conventional methods made it practically impossible. Therefore, it was suggested to create a model based on the neuro-adaptive fuzzy methods.

Thus, as input parameters for the process there have been chosen:

- the diameter;

- the nominal pressure;

- the pipe thickness.

And as an output variable there has been chosen the welding pressure.

From the set of experimental data there was separated a data set for the drive shaft and a data set for verification.

An initial fuzzy model has been built that reflects the system's behavior only to a limited extent. Then, based on the drive shaft and verification data, there was created a refined fuzzy model using artificial neuronal networks and an autolearning process, until the modeling error reached acceptable values. From this stage, it can be said that fuzzy model reflects the real consequences of the process, being also possible to work on it, using characteristic fuzzy methods. 
In order to carry out the activities mentioned above, a specific interface and subprograms was created in the software package Matlab using the Fuzzy Logic Toolbox modules and Neural Network Toolbox software.

Starting from the collected input/output data set, two sets were generated, a first set used as the drive shaft and the second set used as verification data. The two sets of data are shown in tables 2 and 3.

Table 2. Experimental training data set

\begin{tabular}{|c|c|c|c|}
\hline $\begin{array}{c}\text { Nominal pipe } \\
\text { diameter } \\
{[\mathbf{m m}]}\end{array}$ & $\begin{array}{c}\text { Nominal fluid } \\
\text { pressure }[\text { bar }]\end{array}$ & $\begin{array}{c}\text { Pipe wall thickness } \\
{[\mathbf{m m}]}\end{array}$ & $\begin{array}{c}\text { Welding } \\
\text { pressure } \\
{[\text { bar }]}\end{array}$ \\
\hline 75.0000 & 2.5000 & 2.0000 & 2.8000 \\
\hline 75.0000 & 4.0000 & 2.9000 & 3.9000 \\
\hline 75.0000 & 10.0000 & 6.9000 & 8.9000 \\
\hline 90.0000 & 3.2000 & 2.8000 & 4.6000 \\
\hline 90.0000 & 6.0000 & 5.1000 & 8.2000 \\
\hline 90.0000 & 16.0000 & 12.5000 & 18.3000 \\
\hline 110.0000 & 2.5000 & 2.7000 & 5.5000 \\
\hline 110.0000 & 4.0000 & 4.3000 & 8.6000 \\
\hline 110.0000 & 10.0000 & 10.0000 & 18.9000 \\
\hline 125.0000 & 3.2000 & 3.9000 & 8.9000 \\
\hline
\end{tabular}

Table 3. Verification data set

\begin{tabular}{|c|c|c|c|}
\hline $\begin{array}{c}\text { Nominal pipe } \\
\text { diameter } \\
{[\mathbf{m m}]}\end{array}$ & $\begin{array}{c}\text { Nominal fluid } \\
\text { pressure }[\text { bar }]\end{array}$ & $\begin{array}{c}\text { Pipe wall thickness } \\
{[\mathbf{m m}]}\end{array}$ & $\begin{array}{c}\text { Welding } \\
\text { pressure } \\
{[\text { bar }]}\end{array}$ \\
\hline 75.0000 & 3.2000 & 2.4000 & 3.3000 \\
\hline 75.0000 & 6.0000 & 4.3000 & 5.7000 \\
\hline 75.0000 & 16.0000 & 10.3000 & 12.6000 \\
\hline 90.0000 & 2.5000 & 2.2000 & 3.6000 \\
\hline 90.0000 & 4.0000 & 3.5000 & 5.7000 \\
\hline 90.0000 & 10.0000 & 8.1000 & 12.5000 \\
\hline 110.0000 & 3.2000 & 3.5000 & 7.0000 \\
\hline 110.0000 & 6.0000 & 6.3000 & 12.3000 \\
\hline 110.0000 & 16.0000 & 15.1000 & 27.0000 \\
\hline 125.0000 & 2.5000 & 3.1000 & 7.1000 \\
\hline
\end{tabular}

Figure 1 shows the graphical user interface used for determining the mathematical model with the graphical representation of the data on the hard drive. Entering the input and output data into the model's structure is done from two data files (with an extension of "."), which will be then loaded from the computer memory.

It is important to assign the readings into dimensional series or into series of parameters in such a way that the modeling remains valid and the mistakes of interpretation or confusion due to process variables are eliminated. The attributes remain unchanged, regardless of whether the data is for the drive shaft data set or for the verification data set.

To plot the drive shaft data, there was used the graphical symbol "०", while for the representation of the verification data there has been used the symbol "+". Figure 2 shows both data sets. 


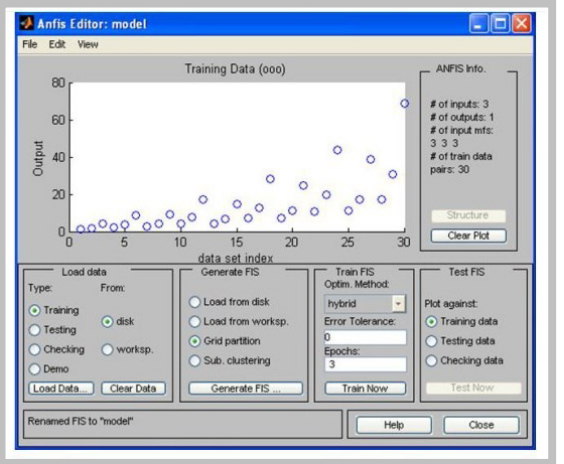

Fig. 1. Graphical interface used for the construction of the neuro-fuzzy model and graphical representation of the data on the hard drive

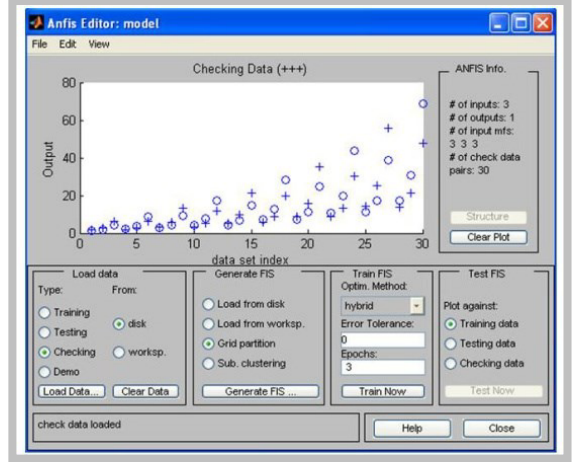

Fig. 2. Graphical representation of the data for the drive shaft and of the verification data.

B. The next step in the construction of the model was to determine a preliminary fuzzy interference structure, based on which to begin the neuro-fuzzy process.

As indicated above, there was chosen a fuzzy model with three input variables (diameter, nominal pressure, thickness) and an output variable (welding pressure).

The preliminary fuzzy structure is shown in figure 3 .

In this preliminary phase, three variation ranges for each variable have been set and the triangular alternative was selected for the affiliation functions.

The distribution of the variation ranges for the diameter input variable and the form of the affiliation functions is shown in figure 4.

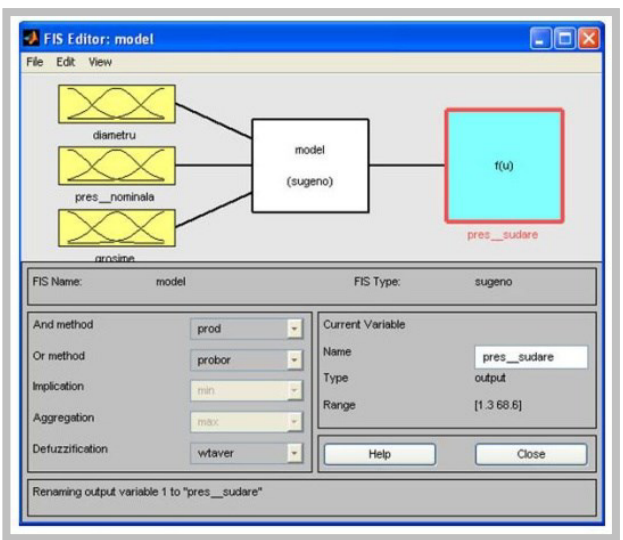

Fig. 3. Preliminary structure of the fuzzy logic system

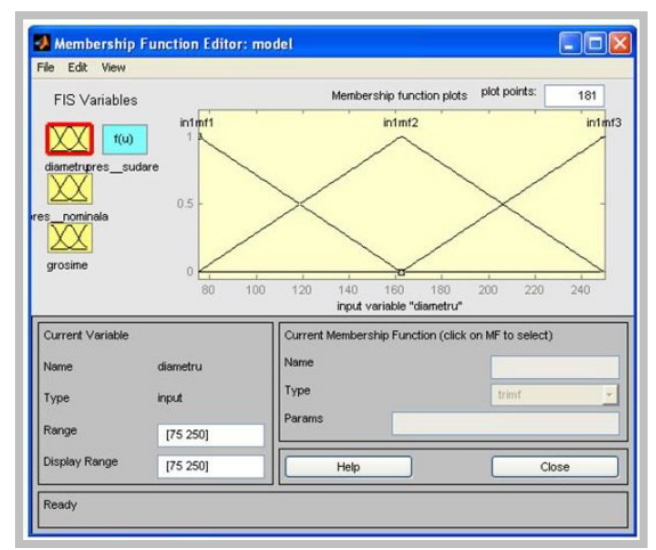

Fig. 4. The function of belonging to the diameter input variable

C. A further stage in the generating the model consisted in unfolding the training process of the neuro-fuzzy system until achieving an acceptable error. The stages of the training process bear the name of ages. For the initial phase of the training process there has been chosen a number of 80 ages. Figure 5 shows the evolution of the training error, which achieves an acceptable minimum after 55 ages of training. After exceeding this value it should be noted that the training error begins to increase, which led to selecting the value of 55 as an optimal number of ages (stages of training). The value of the error after completing the 55 ages is 0.016554 , a value that in this case has been considered as acceptable. 


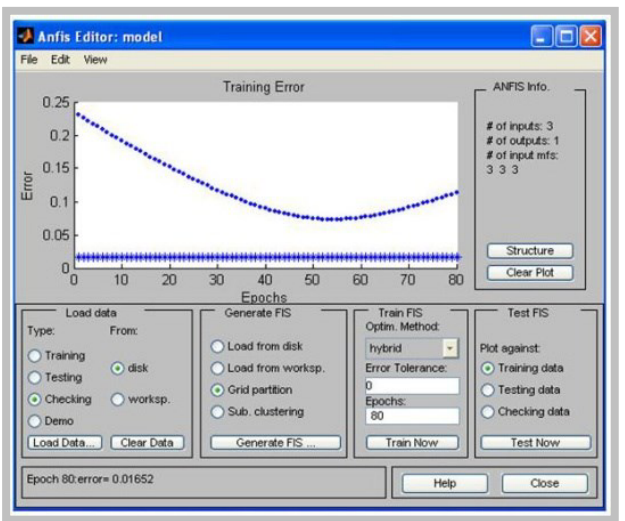

Fig. 5. Evolution of the training error

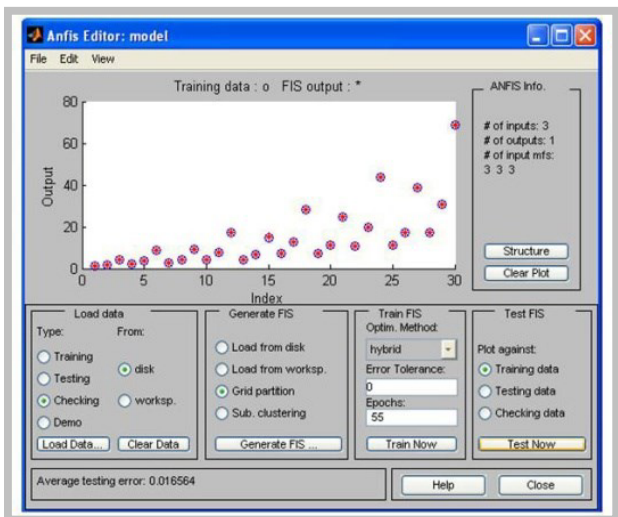

Fig. 6. Predicted data for the model "*" compared to the training data "o",

As training error has been defined the difference between the output value from the sets of training variables, and the output value generated as a result of the fuzzy inference.

D. The next step in the model's construction focused on the quality assessment, through the so-called checking operation. The checking required to compare the values of the experimental output with those obtained by using the neuro-fuzzy model for the same values of the input data.

Figure 6 shows the overlapping of two sets of values, (for displaying the experimental data there was used the symbol "०", while for displaying the data obtained by running the mathematical neurofuzzy model there has been used in the symbol "*").

The tree structure of the neuro-fuzzy system results as shown in figure 7.

A preliminary overview at this stage revealed the existence of 3 variables, 5 levels of the neuronal networks organization, 74 neurons and 27 fuzzy rules.

Figure 8 presents the neuro-fuzzy structure, which at this stage is used to calculate the output for any intermediate values of the variables, in accordance with its intended purpose.

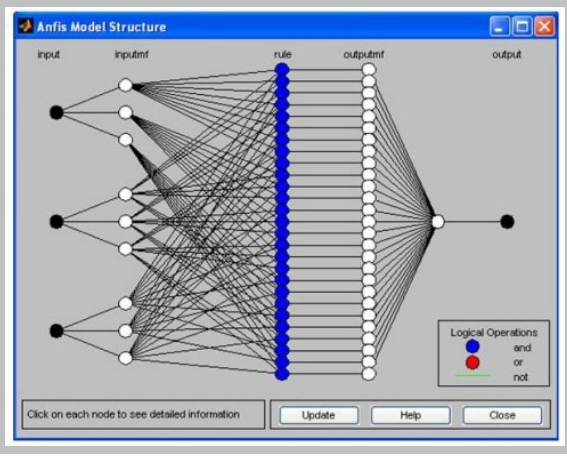

Fig. 7. Tree structure of the fuzzy model

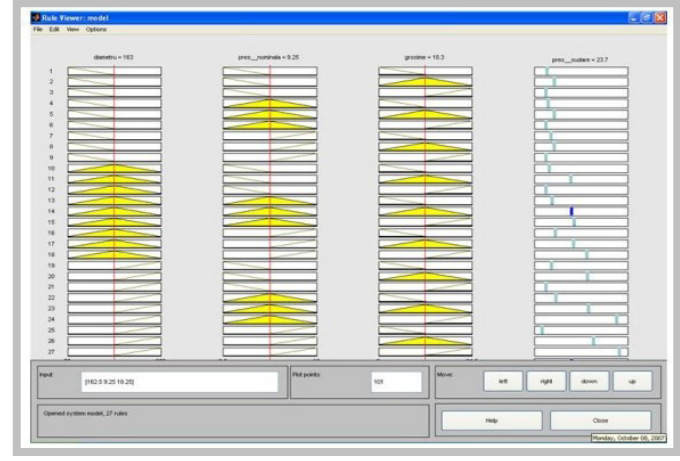

Fig. 8. The neuro-fuzzy model

The main problem which occurs here refers to the share of each input variable in the construction of the mathematical model [4]. A preliminary estimation has revealed that the influence of the variable "nominal pressure" is reduced in comparison to that of the other two, "diameter" and "thickness". However, due to the advanced possibilities of the neuro-fuzzy method used, the variable in question has been taken into account when setting up the model. 
However, based on running the mathematical model, it can be noticed that the value for the variable output is only very slightly influenced by large variations of the input value "nominal pressure". For example, for a variation of the nominal pressure from 3.23 bar to 15.6 bar, the welding pressure varies from 22.4 bar to 29.6 bar.

\subsection{Refining the model}

As a result of the findings described in the previous paragraph, in the model's refining the nominal pressure has been eliminated as an input variable. The running of the neuro-fuzzy system required in this case a number of 35 ages, less than the value of 55 for the previous model with three input variables.

The evolution of the values of the training error (the difference between the output value from the training data set and that obtained by running the neuro-fuzzy model) and of the verification error (the difference between the output value from the verification data set and that obtained by running the neuro-fuzzy model), for the structure of the new model are shown in figure 9.

The structure of the employed fuzzy system was customized by the association of linguistic variables for each input variable. Thus, to the diameter input variable there were associated the variables SMALL, MEDIUM, LARGE, and for the thickness input variable there were associated the linguistic variables LOW, MEDIUM, HIGH.

Figure 10 presents the rules used by the fuzzy system. It should be specified again that generating the rules was carried out in automated mode through the neuro-fuzzy process, but the adaptation and their refining for the studied welding process were made by the authors.

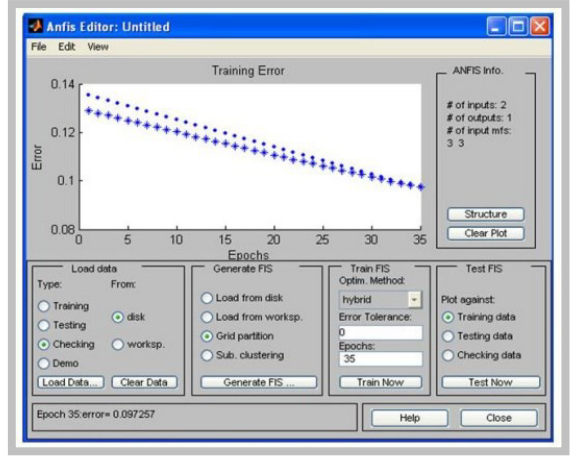

Fig. 9. Evolution of the training and verification errors

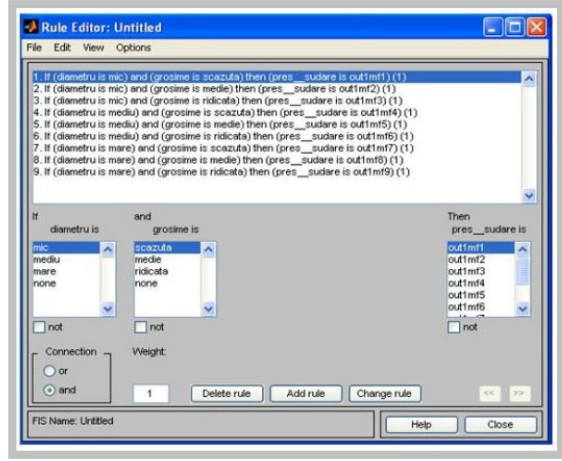

Fig. 10. The fuzzy system rules

Figure 11 presents the adjustment surface of the fuzzy system, which expresses in a graphical form the dependence between the input variables (diameter and thickness) and the output variables (welding pressure).

The neuro-fuzzy model in its final form is shown in figure 12. The model interface allows both the interactive graphical section of the values of the input variables and the accurate input of their values. In both cases the output value is calculated and displayed in real time.

\section{Conclusions}

The generation of the mathematical model for the analyzed welding process has included following stages:

- generation of a preliminary fuzzy model, which combined the user experience with the mathematical system made available by the fuzzy logic; the model's purpose has been to provide some introductory statements on the subsequent modeling strategies; 


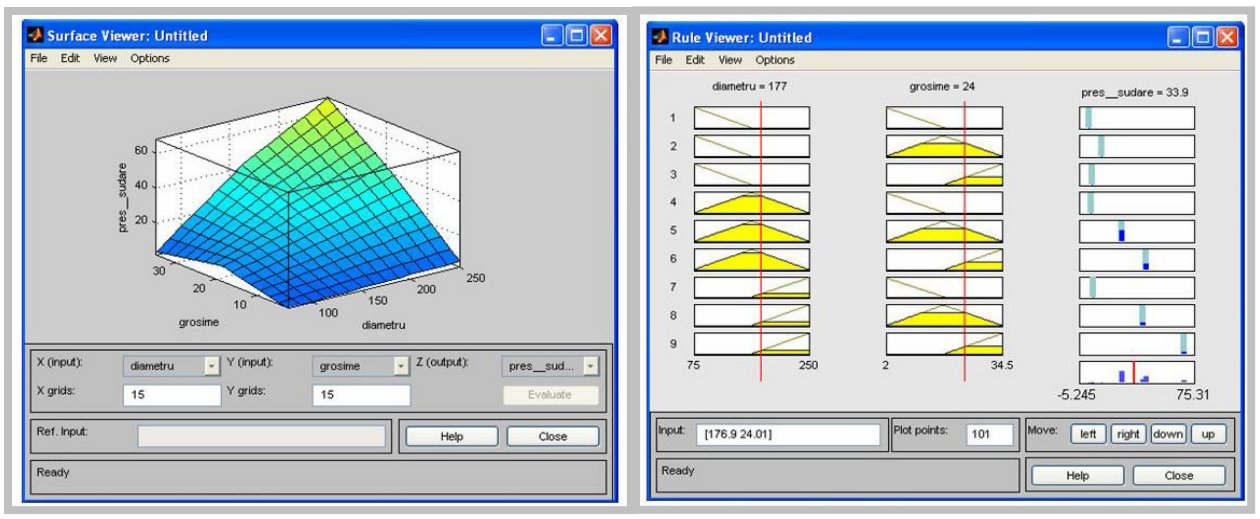

Fig. 11. The fuzzy system adjustment surface

Fig. 12. The neuro-fuzzy model after refining

- creation of a mathematical model whose techniques are based on the neuro-fuzzy concepts, including as an input variable the nominal pressure, although preliminary indications suggested that the size of the output (welding pressure) is very slightly influenced by this; running the developed mathematical model has confirmed this hypothesis;

- refining the previously obtained mathematical model, by eliminating the input variable pressure and adjusting the fuzzy rules and the variation ranges of the quantities, in light of the experience and researches carried out by the authors.

It can be asserted that the mathematical modeling carried out gives the possibility to obtain the correct value of the pressure required for welding, for any pairs of input variables: pipe diameter pipe wall thickness. Also, the proposed model can easily take into account other input variables for the process.

\section{References}

1. D. Conner, EDN 31 (1993)

2. M.H. Hassoun, Fundamentals of Artificial Neural Networks (MIT Press, Cambridge, MA., 1995)

3. M. Jamshidi, N. Vadaiee, T.J. Ross, Fuzzy Logic and Control: Software and Hardware Applications (Prentice-Hall, Englewood Cliffs, 1993)

4. J.F. Agassant, P. Avenas, J-Ph. Sergent, P. Carrean, Polymer Proccessing Principales and Modeling (Hanser Publishers, Munchen, 1991) 